\title{
Ginsenoside ameliorated ventilator-induced lung injury in rats
}

Woo Hyun $\mathrm{Cho}^{1 \dagger}$, Yun Hak Kim${ }^{2+}$, Hye Jin $\mathrm{Heo}^{2}$, Dohyung Kim³ ${ }^{3}$, Tae Won Kwak ${ }^{4}$, Kwang Ho Kim ${ }^{4}$ and Hye Ju Yeo ${ }^{1 *}$ (iD

\begin{abstract}
Background: Ginsenosides have antioxidant and anti-inflammatory features. This study aimed to evaluate the biologic effects of ginsenoside Rb2 pretreatment on ventilator-induced lung injury (VILI) in rats.

Methods: Rats were divided into four groups with 12 rats per group: control; low tidal volume (TV), TV of $6 \mathrm{~mL} / \mathrm{kg}$, VILI, TV of $20 \mathrm{~mL} / \mathrm{kg}$, positive end-expiratory pressure of $5 \mathrm{~cm} \mathrm{H}_{2} \mathrm{O}$, and respiratory rate of 60 breaths per minute for $3 \mathrm{~h}$ at an inspiratory oxygen fraction of 0.21 ; and ginsenosides, treated the same as the VILI group but with $20 \mathrm{mg} /$ $\mathrm{kg}$ intraperitoneal ginsenoside pretreatment. Morphology was observed with a microscope to confirm the VILI model. Wet-to-dry weight ratios, protein concentrations, and pro-inflammatory cytokines in the bronchoalveolar lavage fluid were measured. RNA sequencing of the lung tissues was conducted to analyze gene expression.

Results: High TV histologically induced VILI with alveolar edema and infiltration of inflammatory cells. Ginsenosides pretreatment significantly reduced the histologic lung injury score compared to the VILI group. Wet-to-dry weight ratios, malondialdehyde, and TNF-a in bronchoalveolar lavage fluid were significantly higher in the VILI group and ginsenoside pretreatment mitigated these effects. In the immunohistochemistry assay, ginsenoside pretreatment attenuated the TNF-a upregulation induced by VILI. We identified 823 genes differentially presented in the VILI group compared to the control group. Of the 823 genes, only 13 genes (Arrdc2, Cygb, Exnef, Lcn2, Mroh7, Nsf, Rexo2, Srp9, Tead3, Ephb6, Mvd, Syt14, and Ube216) recovered to control levels in the ginsenoside group.
\end{abstract}

Conclusions: Ginsenosides inhibited the inflammatory and oxidative stress response in VILI. Further studies are required on the 13 genes, including LCN2.

Keywords: Ginsenosides, Ventilator-induced lung injury, Tumor necrosis factor-alpha, LCN2, NGAL

\section{Background}

Acute lung injury (ALI) and acute respiratory distress syndrome (ARDS) are fatal diseases [1]. Mechanical ventilation is essential for overcoming life-threatening hypoxia and hypercapnia. Despite the lifesaving effects,

\footnotetext{
* Correspondence: hjyeo@pusan.ac.kr

†'Woo Hyun Cho and Yun Hak Kim contributed equally to this work.

'Division of Pulmonary, Allergy and Critical Care Medicine, Department of Internal Medicine, Research Institute for Convergence of Biomedical Science and Technology, Pusan National University Yangsan Hospital, Geumo-ro 20, Beomeo-ri, Mulgeum-eup, Yangsan-si, Gyeongsangnam-do 626-770, Republic of Korea

Full list of author information is available at the end of the article
}

mechanical ventilation may induce or deteriorate lung injury, a state noted as ventilator-induced lung injury (VILI) [2]. VILI involves neutrophil activation, high permeability pulmonary edema, increased vascular permeability, and inflammation [2]. Hyperinflated lung caused by mechanical stretch induces a biochemical response, which facilitates neutrophils and inflammatory signals [3]. Increased inflammatory mediators such as cytokines cause complex interactions [4]. The potential mechanisms regarding the transition from mechanical stress to a biochemical reaction have been widely evaluated.

(c) The Author(s). 2020 Open Access This article is licensed under a Creative Commons Attribution 4.0 International License, which permits use, sharing, adaptation, distribution and reproduction in any medium or format, as long as you give appropriate credit to the original author(s) and the source, provide a link to the Creative Commons licence, and indicate if changes were made. The images or other third party material in this article are included in the article's Creative Commons licence, unless indicated otherwise in a credit line to the material. If material is not included in the article's Creative Commons licence and your intended use is not permitted by statutory regulation or exceeds the permitted use, you will need to obtain permission directly from the copyright holder. To view a copy of this licence, visit http://creativecommons.org/licenses/by/4.0/ The Creative Commons Public Domain Dedication waiver (http://creativecommons.org/publicdomain/zero/1.0/) applies to the data made available in this article, unless otherwise stated in a credit line to the data. 
Despite the intense interest in a therapeutic target for treating VILI, no available pharmacologic option exists.

Panax ginseng is the most commonly used herbal medicine since ancient times. Most of the biological functions of Panax ginseng come from ginsenosides [5]. Ginsenosides have anti-inflammatory activities achieved by inhibiting the production of pro-inflammatory cytokines and inhibiting the actions of inflammatory signaling pathways $[6,7]$. In particular, ginsenoside $\mathrm{Rb} 2(\mathrm{GnRb} 2)$ significantly inhibits the transcriptional activity of NF-kB $[8,9]$. Multiple studies have been conducted to understand the role and mechanisms of ginsenosides in a variety of diseases, including diabetes mellitus, cardiovascular disease, and cancers [10-13]. However, no related study has showed the effects of GnRb2 pretreatment on VILI. Considering the anti-inflammatory and antioxidant function, we hypothesized that $\mathrm{GnRb} 2$ will have protective effects in VILI. To verify this hypothesis, we ventilated rats with high tidal volume (TV) and evaluated the effects of GnRb2 in attenuating VILI in rats. Furthermore, we conducted a gene expression profiling analysis of the lung tissues to understand the underlying mechanism and identify potential therapeutic targets.

\section{Methods}

\section{Materials}

Korean red ginseng extract was manufactured by the Korea Ginseng Corporation (Seoul, Korea). GnRb2 was purified as described by Kitagawa et al. [14], and the purity was higher than $99.9 \%$.

\section{Animal preparation}

Eight-week-old Sprague-Dawley rats weighing 250-300 g were used. All surgeries were performed under ketamine anesthesia given intraperitoneally $(100 \mathrm{mg} / \mathrm{kg}$; Eurovet Animal Health BV, Bladel, the Netherland). The rats were randomly divided into four groups ( $N=12$ per group): control, low TV, VILI, and GnRb2. The control group underwent tracheostomy after anesthesia and breathed spontaneously without mechanical ventilation (MV). The low TV group was ventilated with an animal ventilator (model 683) (Harvard Apparatus, Holliston, MA, USA) using a TV of $6 \mathrm{~mL} /$ $\mathrm{kg}$, positive end-expiratory pressure of $5 \mathrm{cmH}_{2} \mathrm{O}$, and respiratory rate of 60 breaths per minute for $3 \mathrm{~h}$ at an inspiratory oxygen fraction of 0.21 . The VILI group was ventilated with an animal ventilator (model 683) (Harvard Apparatus, Holliston, MA, USA) using a tidal volume of $20 \mathrm{~mL} / \mathrm{kg}$. The VILI group has used the ventilator the same way as the low TV group except for high TV. The GnRb2 group was treated with $20 \mathrm{mg} / \mathrm{kg} \mathrm{GnRb2}$ intraperitoneally $5 \mathrm{~h}$ before ventilator initiation. And the GnRb2 group was treated the same as the VILI group. All rats were sacrificed $3 \mathrm{~h}$ after tracheostomy.

\section{Morphologic changes in the lung tissues}

For histological evaluation, the lung specimen was fixed with the same condition with the lung expanded. Histological examination was performed on the right upper lobe of the lung as described in previous studies $[15,16]$. Five nonoverlapping fields of lung sections were examined by two pathologists (Knotus Co. Ltd., Incheon, Republic of Korea) who were blinded to the group of the rat. Lung injury was evaluated as described in [15] for (I) alveolar capillary congestion; (II) hemorrhage; (III) infiltration of neutrophils into the airspace, vessel wall, or alveolar wall; and (IV) alveolar wall thickness/hyaline membrane formation. Severity were scored as follows: 0 (normal), 1 (mild, $<25 \%$ ), 2 (moderate, 25-50\%), 3 (severe, 50-75\%), 4 (very severe, > 75\%). The lung injury score is the sum of all scores for each item.

\section{Assess of pulmonary edema}

We evaluated pulmonary edema using wet-to-dry weight ratios. We sacrificed rats by arterial bleeding after $3 \mathrm{~h}$ ventilation and removed the entire lungs (six rats in each group). The lungs were weighed immediately (wet weight) and then placed in an oven at $60^{\circ} \mathrm{C}$ for $72 \mathrm{~h}$. After drying, the lungs were weighed again (dry weight). The wet-to-dry weight ratio was defined as the (wet weight-dry weight)/dry weight.

\section{Measuring protein concentrations and cytokines}

Bronchoalveolar lavage fluid (BALF) was acquired from the left upper lung as described previously [16]. Myeloperoxidase (MPO) and malondialdehyde (MDA) in BALF were measured in a blinded fashion using the appropriate MPO (ab105136, Abcam, Cambridge, UK) or MDA (ab118970, Abcam, Cambridge, UK) kits (Supplement 1). Cytokines were measured in a blinded fashion, using rat-specific ELISA kits (Supplement 1).

\section{Immunohistochemical staining of TNF-a}

TNF- $\alpha$ staining was performed on serial sections from six specimens per group. Tissues were incubated overnight at $4{ }^{\circ} \mathrm{C}$ with a rabbit polyclonal antibody against TNF-a (Abcam, Cambridge, UK). Primary antibody incubation was followed by the addition of donkey anti-rabbit IgG antibody incubation (Abcam, Cambridge, UK).

\section{RNA sequencing}

We extracted RNA using formalin-fixed, paraffinembedded (FFPE) lung tissues. RNA purity was controlled by analyzing $1 \mu \mathrm{L}$ of total RNA extract on a NanoDrop 8000 spectrophotometer. Total RNA integrity was confirmed using an Agilent Technologies 2100 Bioanalyzer with an RNA Integrity Number value. Total RNA sequencing libraries were prepared according to the manufacturer's instructions (Illumina Truseq Stranded Total RNA Sample Prep kit with Ribo-zero human). Total RNA was subjected 
to ribosomal RNA depletion, with Ribo-zero human reagent, using biotinylated probes that selectively bind rRNA species. Following purification, the rRNA-depleted total RNA was fragmented into small pieces using divalent cations under elevated temperature. The cleaved RNA fragments were copied into first-strand cDNAs using reverse transcriptase and random primers. This was followed by second-strand cDNA synthesis using DNA polymerase I and RNase $\mathrm{H}$. A single "A" base was added to the cDNA fragments, which were subsequently ligated with the adapter. The products were purified and enriched, using PCR to create the final cDNA library. The quality of the amplified libraries was checked by capillary electrophoresis (Bioanalyzer, Agilent). After QPCR using SYBR Green PCR Master Mix (Applied Biosystems), we pooled index-tagged libraries in equimolar amounts. Cluster generation occurred in the flow cell on the cBot automated cluster generation system (Illumina). Then, the flow cell was loaded into the Novaseq 6000 sequencing system (Illumina), and sequencing was conducted with a $2 \times 100$ bp read length.

\section{Statistical analysis}

All experimental data are showed as the mean \pm SEM. One-way repeated measure ANOVAs (RM ANOVA) (within-subject factor: treatment) were run, followed by Tukey's or Scheffe post hoc tests to evaluate treatment effects. A $P$ value of $<0.05$ was regarded as significant. Statistical analyses were conducted using $\mathrm{R}$ software version 3.5.1.

\section{Results}

Pathologic changes in the lung tissue

Histological examination of the lung tissues in the control group showed complete pulmonary alveoli structures, no alveolar edema, no significant inflammatory cell infiltration, and no evidence of hyaline membrane formation (Fig. 1a). The low TV group showed mild interstitial neutrophil infiltration (Fig. 1b). The VILI group showed interstitial perivascular edema, intraalveolar and interstitial neutrophil infiltration, alveolar hemorrhage, and hyaline membrane formation (Fig. 1c). The lungs of the GnRb2 group presented with only

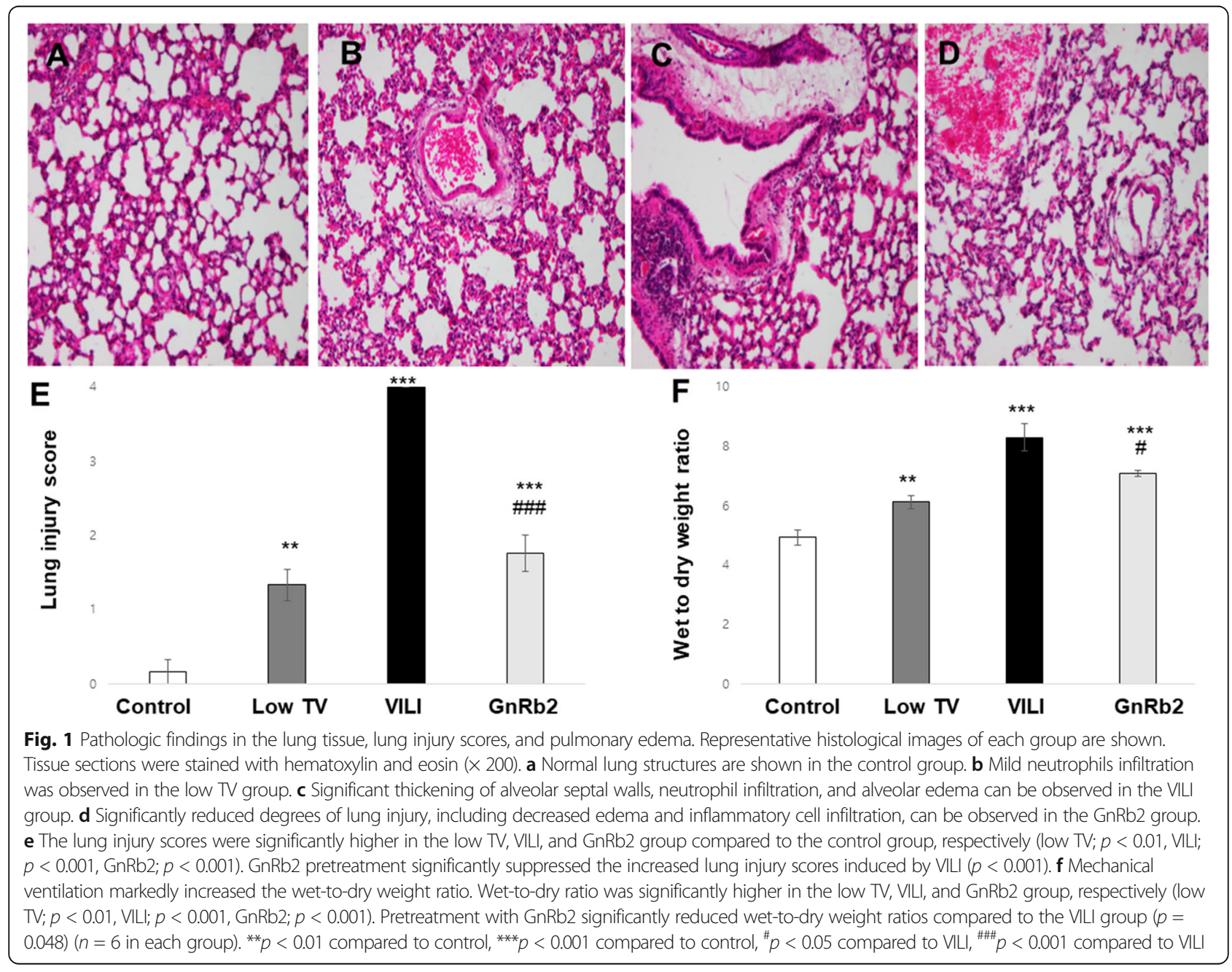


minor signs of alveolar edema and inflammatory cell infiltration (Fig. 1d).

\section{Lung injury score}

The lung injury scores were significantly higher in the low TV, VILI, and GnRb2 group compared to the control group, respectively (low TV; $p<0.01$, VILI; $p<0.001$, GnRb2; $p<0.001)$. GnRb2 pretreatment significantly attenuated the increased lung injury score induced by VILI $(p<0.001)$ (Fig. 1e).

\section{Assessment of pulmonary edema}

Wet-to-dry ratio was significantly higher in the low TV, VILI, and GnRB2 group, respectively (low TV; $p<0.01$, VILI; $p<0.001$, GnRb2; $p<0.001$ ). Wet-to-dry weight ratios were reduced in the $\mathrm{GnRb} 2$ group compared to the VILI group $(p<0.05)$ (Fig. 1f).

\section{Cytokine levels in BALF}

The TNF- $\alpha$ concentration in BALF was significantly higher in the low TV and VILI group compared to the control group (low TV; $p=0.004$, VILI; $p=0.004$ ). The TNF- $\alpha$ concentration in the GnRb2 group was decreased compared to the VILI group ( $p=0.014$ ) (Fig. 2a). IL-8 also increased significantly in the VILI compared to the control group ( $p<0.001$, Fig. $2 c)$. However, IL-8 in the
GnRb2 group did not significantly decrease compared to the VILI group. As well, IL-6 and IL-1 $\beta$ did not differ between the groups (Fig. 2b, d).

\section{The MPO activity in BALF}

The BALF MPO activity was significantly higher in the VILI group compared to the control group $(p=0.011)$. But there was no significant difference in BALF MPO activity between the VILI and GnRb2 groups (Fig. 2e).

\section{Oxidative stress in the lung tissue}

The MDA concentration in BALF was significantly increased in the VILI group compared to the control group ( $p=0.024)$. MDA was decreased in the GnRb2 group compared to the VILI group, but it was not statistically significant ( $p=0.234)$ (Fig. 2f).

\section{Differentially expressed genes}

Results of the gene array experiments are presented as fold changes between the three groups: control, VILI, and $\mathrm{GnRb2}$. Comparisons were performed step by step between the three groups (control vs VILI, VILI vs GnRb2). A heatmap of genes that differed significantly between the three groups is shown in Fig. 3 (a, upregulated genes; b, downregulated genes). We identified 823 differentially expressed genes in the VILI group

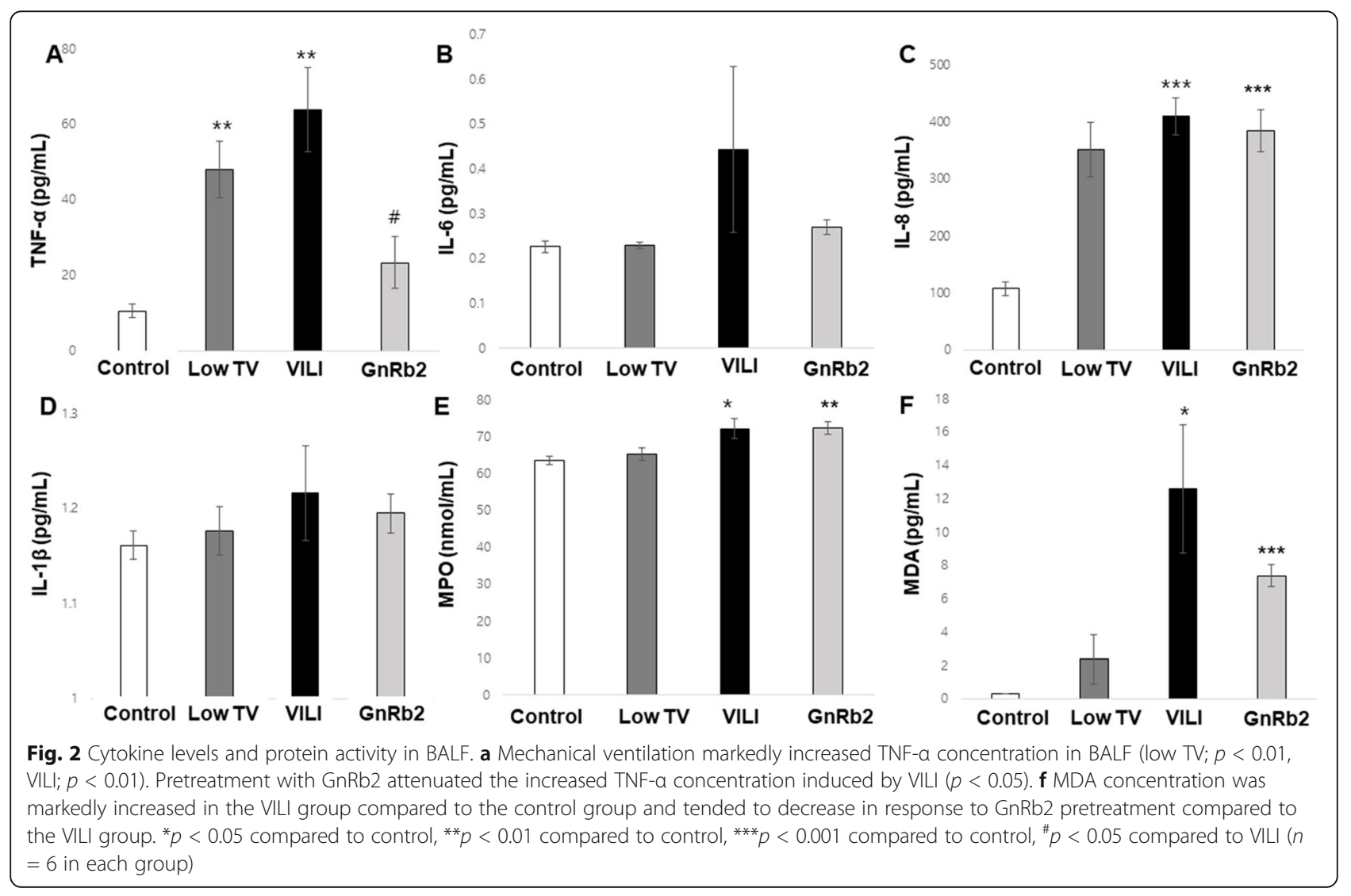


A.upregulated

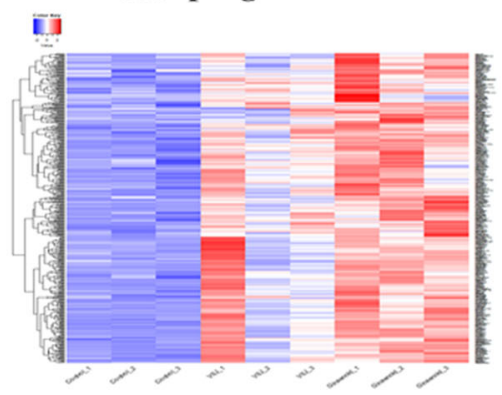

C. Up-regulated genes

Control vs VILI (431)

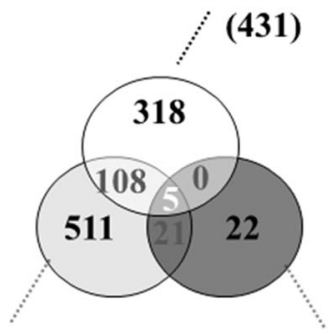

Control vs GnRb2 VILI vs GnRb2 (645)
(48)
B. downregulated

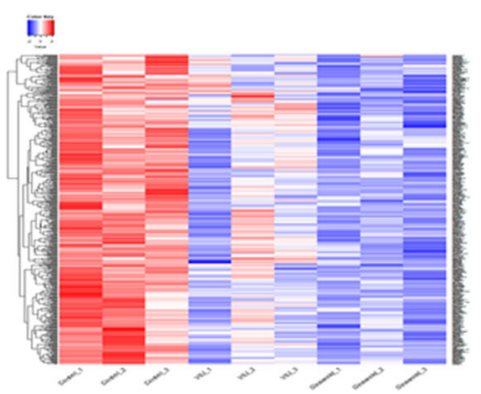

D. Down-regulated genes

Control vs VILI

(392)

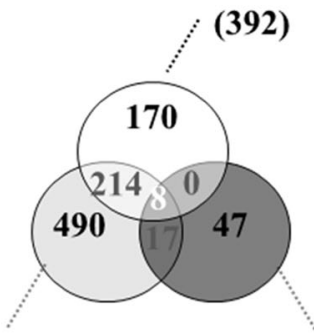

Control vs GnRb2

(729)

\section{VILI vs GnRb2}

(72)

Fig. 3 Comparative heatmap depiction of differential gene expression and differentially expressed genes between three groups. a Heatmap of upregulated genes. $\mathbf{b}$ Heatmap of downregulated genes. $\mathbf{c}$, $\mathbf{d}$ The Venn diagram shows the number of overlapping altered genes between the control, VILI, and GnRb2 groups

compared to the control group, including 431 upregulated and 392 downregulated genes (Fig. 3c, d). The inflammatory cytokines such as TNF- $\alpha(p=0.084), \mathrm{IL}-1 \beta$ $(p=0.352)$, and IL-18 ( $p=0.725)$ were increased in the VILI group compared to the control, but not significant. Of the 431 genes that were upregulated in the VILI group, only 9 genes (Arrdc2, Cygb, Exnef, Lcn2, Mroh7, Nsf, Rexo2, Srp9, and Tead3) recovered to control levels in the GnRb2 group (Fig. 4). Of the 392 genes that were downregulated in the VILI, only 4 genes (Ephb6, Mvd, Sytl4, and Ube2l6) recovered to control levels in the GnRb2 group (Fig. 5).

\section{Immunohistochemical expression of TNF-a}

Representative images showing the immunohistochemical expression of TNF- $\alpha$ in the three groups are shown in Fig. 6. Immunohistochemical staining of TNF- $\alpha$ was more intense in the VILI group compared to the control group. Immunohistochemical staining for TNF- $\alpha$ was weaker in the GnRb2 group compared to the VILI group.

\section{Discussion}

Our study shows the anti-inflammatory effects of $\mathrm{GnRb2}$, which is commonly used as an herbal medicine, in an experimental rat model of VILI. Intraperitoneal injection of $\mathrm{GnRb} 2$ alleviated the biochemical and histologic changes induced by VILI. These anti-inflammatory effects of GnRb2 may be mediated via the suppression of TNF- $\alpha$ activation. Among the upregulated or downregulated genes in the VILI group, only 13 genes $(A r r d c 2$, Cygb, Exnef, Lcn2, Mroh7, Nsf, Rexo2, Srp9, Tead3, Ephb6, Mvd, Sytl4, and Ube2l6) recovered to control levels in the GnRb2 group. Of the 13 genes, lipocalin 2 (LCN2) is a potential downstream target of TNF- $\alpha$ leading to the protective effect of GnRb2 in the VILI model.

The anti-inflammatory effects of Ginseng derivatives have been demonstrated in various experimental models, mainly cell studies $[17,18]$. However, the pathogenic mechanisms of the anti-inflammatory effects are not fully understood and the effects of Ginseng derivatives in a VILI model have not been studied. In this study, pretreatment with GnRb2 decreased wet-to-dry weight ratio, MDA concentration, and histologic lung injury score in a rat model of VILI. These results support our hypothesis that GnRb2 protects the lung from VILI and prevents the progress of pulmonary edema; the typical pathological damage induced by VILI is mitigated by GnRb2.

In our study, GnRb2 pretreatment decreased TNF- $\alpha$ concentration in BALF and immunohistochemistry 


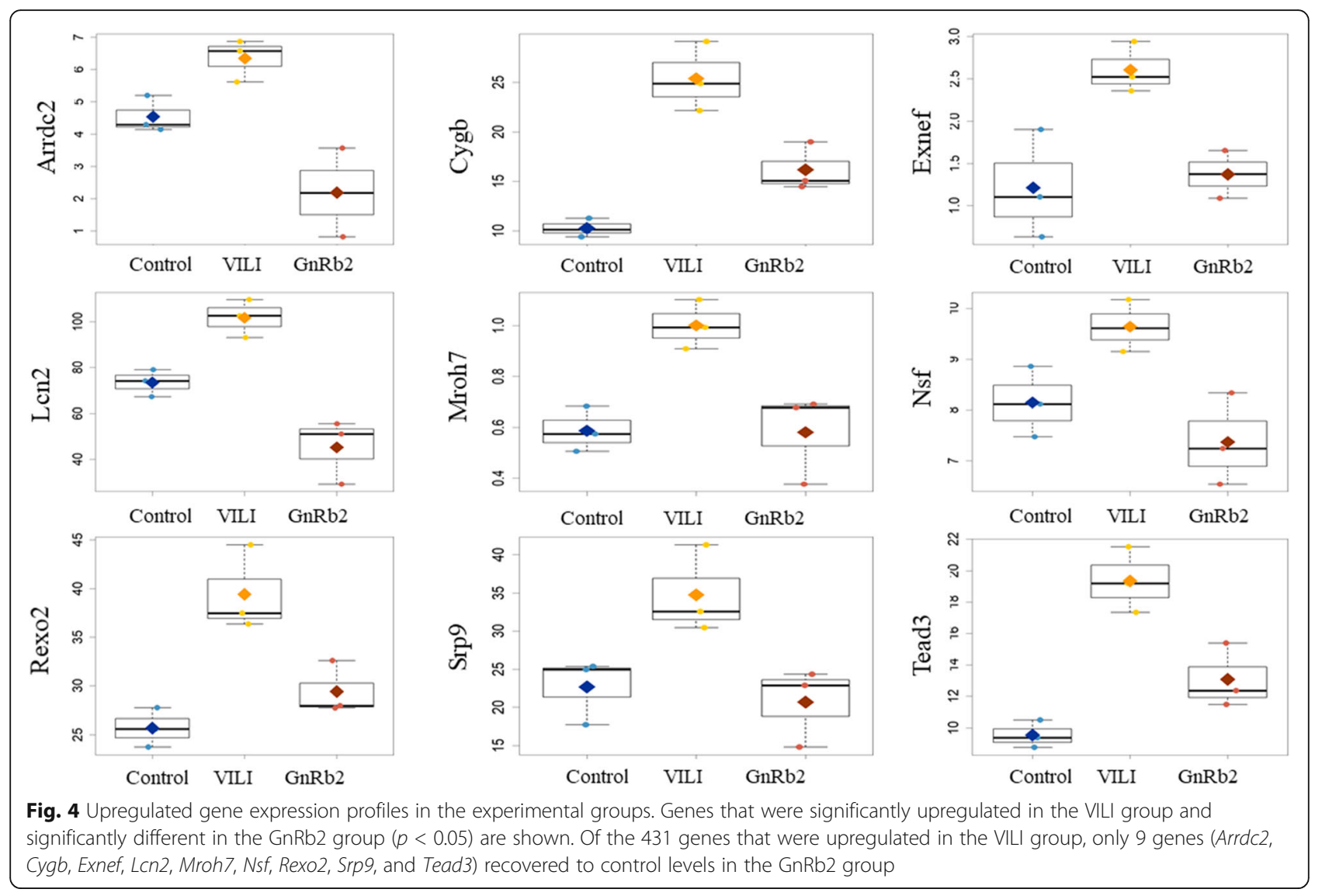

staining of TNF- $\alpha$. Previously, GnRb2 has been shown to inhibit TNF- $\alpha$ production and/or inhibit the transcriptional activity of NF-kB, but the mechanism is not well understood $[8,9,19]$. Generally, NF-kB exists as inactive NF-kB dimers in the cytosol of unstimulated cells and is activated by phosphorylation of the inhibitor of NF$k B$ proteins (IkBs) [20]. Various cellular stimuli such as TNF- $\alpha$ lead to degradation of IkBs via IKB kinase (IKK) complex, which is responsible for IkB phosphorylation. Previously, Wu et al. reported that GnRb2 inhibits TNF- $\alpha$ production through NF-kB inhibition by inhibition of phosphorylation of IkBa [21]. Unfortunately, gene expression profiling of this study did not find any difference in the level of IkBs and IKK among the three groups. As well, there were no significant differences in gene expression profiling of the promoter genes of TNF- $\alpha$ between the three groups. Only a significant change in the LCN2 gene, one of the downstream changes of TNF- $\alpha$, was observed, suggesting a potential role of ginsenosides through the TNF- $\alpha$-LCN2 pathway. LCN2 is derived from specific neutrophil granules and is widely participated in inflammatory and immune responses as a marker of neutrophil activation [22]. In a previous study, LCN2 expression increased in VILI animal models in accordance with the degree of lung injury [23]. LCN2 activation can be mediated by pro- inflammatory cytokines, such as IL-1 $\beta$ and TNF- $\alpha$ [24-26]. In this study, we failed to reveal whether the GnRb2 affect the upregulation of LCN2 through NF-kB or an alternative pathway. Given that there is no significant difference in the level of NF-kB among gene expression profiling between the three groups, there is a possibility of the upregulation of LCN2 through an alternative pathway. Further researches are required to verify a potential alternative pathway suppressing the level of TNF- $\alpha$ by GnRb2 in a VILI model.

To date, of the remaining 12 genes, there are no reports associated with VILI. All of 13 genes are potentially associated with crosstalk between VILI and the therapeutic effect of GnRb2. However, we could not find the additional biochemical evidence to convince the rest of the 12 genes except LCN2 were related to VILI and GnRb2. Nethylmaleimide sensitive factor (NSF) is related to the secretion of inflammatory mediators from pulmonary microvascular endothelial cells and vascular instability [27]. Mechanical stress in the pulmonary vasculature is one of the mechanisms causing pathophysiologic changes in VILI [28]. In this study, GnRb2 reduces NSF expression in VILI, suggesting that the protective effect of GnRb2 might be associated with the endothelial injury. Further research is required on other genes, including NSF. 

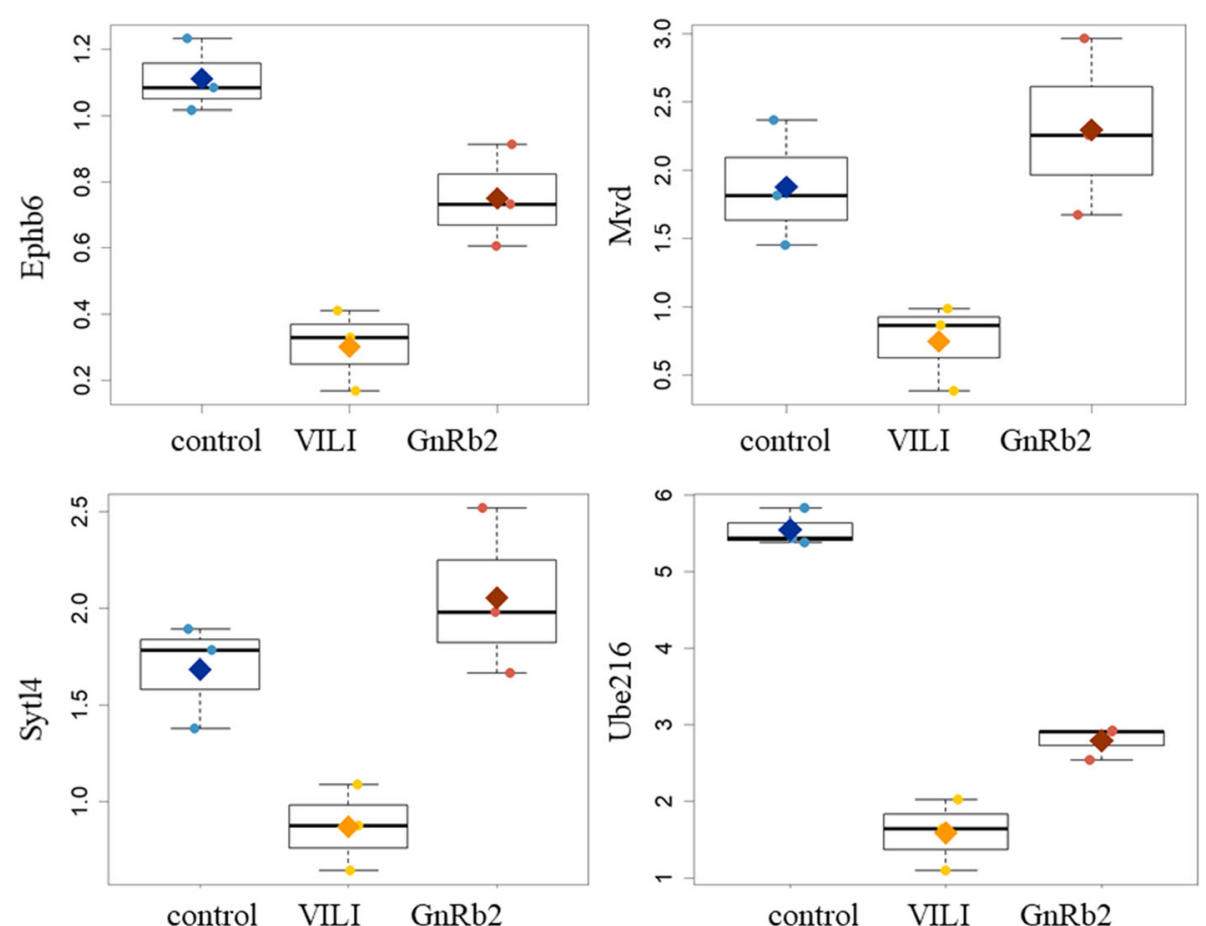

Fig. 5 Downregulated gene expression profiles in the experimental groups. Genes that were significantly downregulated in the VILI group and significantly different in the GnRb2 group $(p<0.05)$ are shown. Of the 392 genes that were downregulated in the VILI group, only 4 genes (Ephb6, Mvd, Syt/4, and Ube216) recovered to control levels in the GnRb2 group

This study has several limitations including a small sample size and uncertainty about the fidelity of FFPE samples. First, there are concerns regarding data quality and interpretation of RNA derived from FFPE. FFPE tissue processing and sample storage are known to result in highly degraded RNA, which may limit gene detection and introduces sequencing artifacts. However, as recent technical advances, extracting RNA from FFPE tissues does not affect the overall quality of results [29-33]. The variable reproducibility of gene signatures probably relates to the reliability of the individual gene selected and possibly to the algorithm. Second, this study did not fully confirm the cellular response in protein level, only found the significant changes of TNF- $\alpha$ through the

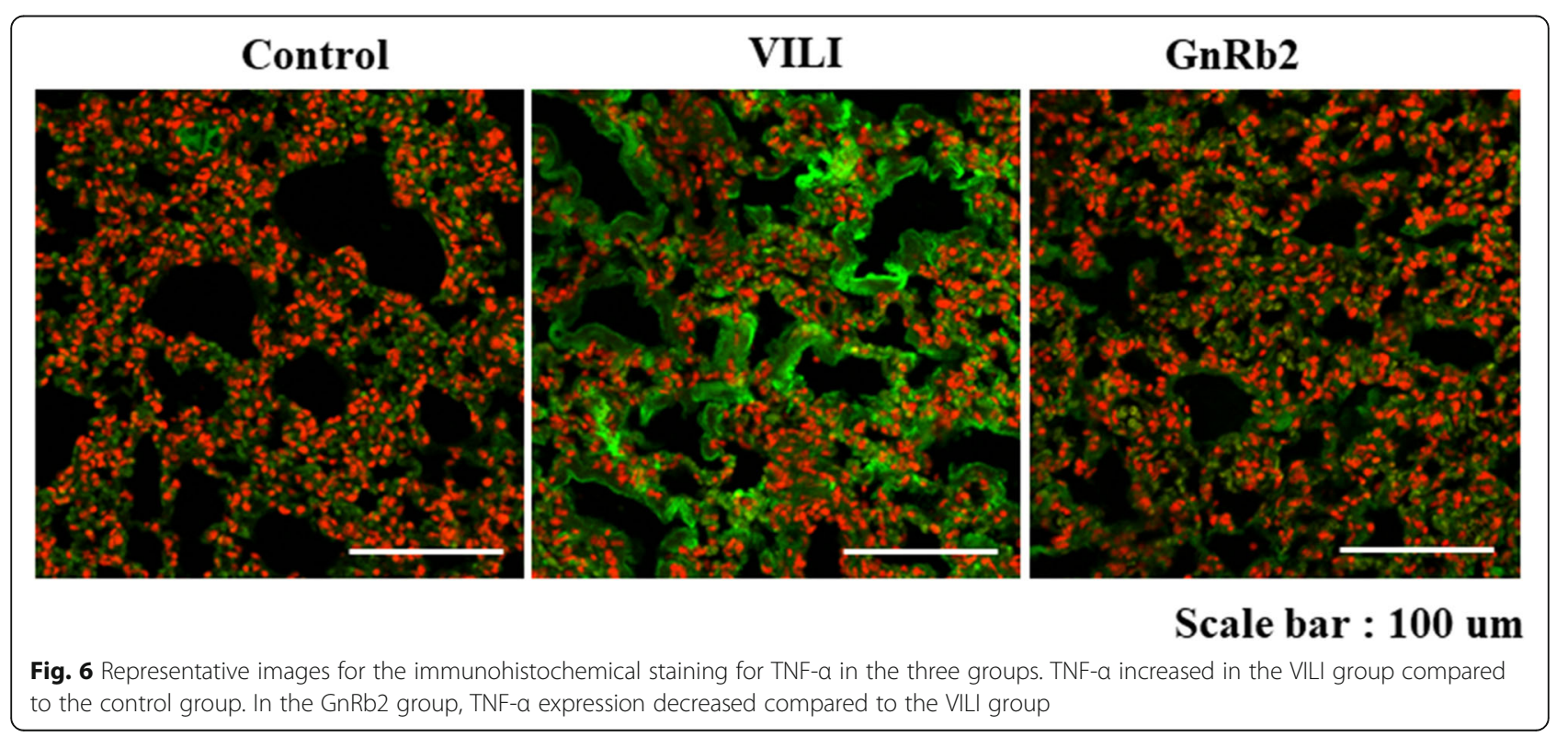


ELISA test and IHC stain. Although this is partially convincing evidence, GnRb2 was associated with TNF- $\alpha$ reduction and its downstream, LCN2. Based on these pilot results, further studies are required to verify the therapeutic mechanism of GnRb2 on TNF- $\alpha$ level and the significance of the 13 genes in a VILI and GnRb2 treatment. Third, the arterial oxygen tension was not measured in our VILI model. Therefore, this finding should be cautiously interpreted to apply real clinical practice due to a lack of physiologic benefits regarding oxygenation improvement. Further scientific evidence aiming at physiologic benefit should be preceded to a future clinical trial.

\section{Conclusions}

In conclusion, our study showed that the GnRb2 pretreatment attenuates VILI, including inflammation and pulmonary edema. Thirteen genes were differentially expressed in response to GnRb2 pretreatment in VILI. Among the differentially expressed genes, the effect of $\mathrm{GnRb} 2$ on lung injury is associated with the downregulation of LCN2 by TNF- $\alpha$ inhibition. GnRb2 is a potential therapeutic agent for treating or preventing VILI through inhibition of the TNF- $\alpha$ signaling pathway.

\section{Supplementary Information}

The online version contains supplementary material available at https://doi. org/10.1186/s40560-020-00509-5.

Additional file 1:. Supplement 1. ELISA kits used in this study.

\section{Abbreviations}

ALI: Acute lung injury; ARDS: Acute respiratory distress syndrome; VILI: Ventilator-induced lung injury; GnRb2: Ginsenoside Rb2; MV: Mechanical ventilation; BALF: Bronchoalveolar lavage fluid; MPO: Myeloperoxidase; MDA: Malondialdehyde; LCN2: Lipocalin 2

\section{Acknowledgements}

None

\section{Authors' contributions}

Conception and design: Dohyung Kim. Administrative support: Hye Ju Yeo. Provision of the study materials: Tae Won Kwak and Kwang Ho Kim. Collection and assembly of the data: Hye Jin Heo and Yun Hak Kim. Data analysis and interpretation: Yun Hak Kim and Woo Hyun Cho. The authors read and approved the final manuscript.

\section{Funding}

This study was supported by the Research Institute for Convergence of Biomedical Science and Technology (30-2018-003), Pusan National University Yangsan Hospital.

\section{Availability of data and materials}

The datasets used and/or analyzed during the current study are available from the corresponding author on reasonable request.

Ethics approval and consent to participate

Our experiments were permitted by the Animal Ethics Committee of the Gyeongbuk Technopark (R17001).

\section{Consent for publication}

Not applicable

\section{Competing interests}

The authors have no conflicts of interest to declare.

\section{Author details}

${ }^{1}$ Division of Pulmonary, Allergy and Critical Care Medicine, Department of Internal Medicine, Research Institute for Convergence of Biomedical Science and Technology, Pusan National University Yangsan Hospital, Geumo-ro 20, Beomeo-ri, Mulgeum-eup, Yangsan-si, Gyeongsangnam-do 626-770, Republic of Korea. ${ }^{2}$ Department of Anatomy and Department of Biomedical Informatics, School of Medicine, Pusan National University, Busan, Republic of Korea. ${ }^{3}$ Department of Thoracic and Cardiovascular surgery, Pusan National University Yangsan Hospital, Yangsan-si, Republic of Korea. ${ }^{4}$ Medical Convergence Materials Commercialization Center, GyeongBuk TechnoPark, Gyeongbuk, Republic of Korea.

Received: 3 August 2020 Accepted: 15 November 2020

Published online: 23 November 2020

\section{References}

1. Bellani G, Laffey JG, Pham T, et al. Epidemiology, patterns of care, and mortality for patients with acute respiratory distress syndrome in intensive care units in 50 countries. JAMA. 2016;315:788-800. https://doi.org/10.1001/jama.2016.0291.

2. Hegeman MA, Hennus MP, Cobelens PM, et al. Dexamethasone attenuates VEGF expression and inflammation but not barrier dysfunction in a murine model of ventilator-induced lung injury. PLoS One. 2013;8:e57374.

3. Silva PL, Negrini D, Rocco PR. Mechanisms of ventilator-induced lung injury in healthy lungs. Best Pract Res Clin Anaesthesiol. 2015;29:301-13.

4. Curley GF, Laffey JG, Zhang H, et al. Biotrauma and ventilator-induced lung injury: clinical implications. Chest. 2016;150:1109-17.

5. Yun TK, et al. J Korean Med Sci. 2001;16(Suppl(Suppl)):S3-5. https://doi.org/ 10.3346/jkms.2001.16.S.S3.

6. Kim JH, Yi YS, Kim MY, Cho JY. Role of ginsenosides, the main active components of Panax ginseng, in inflammatory responses and diseases. J Ginseng Res. 2017;41:435-43.

7. Lee DCW, Lau ASY. Effects of Panax ginseng on tumor necrosis factor-amediated inflammation: a mini-review. Molecules. 2011;16:2802-16.

8. Cho K, Song SB, Tung NH, Kim KE, Kim YH. Inhibition of TNF-a-mediated NF-KB transcriptional activity by dammarane-type ginsenosides from steamed flower buds of Panax ginseng in HepG2 and SK-Hep1 cells. Biomol Ther (Seoul). 2014;22:55-61. https://doi.org/10.4062/biomolther.2013.096.

9. Song SB, Tung NH, Quang TH, Ngan NT, Kim KE, Kim YH. Inhibition of TNFa-mediated NF-kB transcriptional activity in HepG2 cells by dammaranetype saponins from Panax ginseng leaves. J Ginseng Res. 2012;36:146-52. https://doi.org/10.5142/jgr.2012.36.2.146.

10. Mohanan P, Subramaniyam S, Mathiyalagan R, Yang DC. Molecular signaling of ginsenosides Rb1, Rg1, and Rg3 and their mode of actions. J Ginseng Res. 2018;42:123-32. https://doi.org/10.1016/j.jgr.2017.01.008.

11. Bai L, Gao J, Wei F, Zhao J, Wang D, Wei J. Therapeutic potential of Ginsenosides as an adjuvant treatment for diabetes. Front Pharmacol. 2018; 9:423. Published 2018 May 1. doi:https://doi.org/10.3389/fphar.2018.00423.

12. Chen T, Li B, Qiu Y, Qiu Z, Qu P. Functional mechanism of Ginsenosides on tumor growth and metastasis. Saudi J Biol Sci. 2018;25:917-22. https://doi. org/10.1016/j.sjbs.2018.01.012.

13. Sun $Y$, Liu $Y$, Chen $K$. Roles and mechanisms of ginsenoside in cardiovascular diseases: progress and perspectives. Sci China Life Sci. 2016; 59:292-8. https://doi.org/10.1007/s11427-016-5007-8.

14. Kitagawa I, Yoshikawa M, Yoshihara M, Hayashi T, Taniyama T. Chemical studies of crude drugs (1). Constituents of ginseng radix rubra. Yakugaku Zasshi. 1983;103:612-22

15. Belperio JA, Keane MP, Burdick MD, et al. Critical role for CXCR2 and CXCR2 ligands during the pathogenesis of ventilator-induced lung injury. J Clin Invest. 2002;110:1703-16.

16. Kim D-H, Chung JH, Son BS, Kim YJ, Lee SG. Effect of a neutrophil elastase inhibitor on ventilator-induced lung injury in rats. J Thorac Dis. 2014;6:1681-9.

17. Kim DH, Kim DW, Jung BH, Lee JH, Lee H, Hwang GS, et al. Ginsenoside Rb2 suppresses the glutamate-mediated oxidative stress and neuronal cell death in HT22 cells. J Ginseng Res. 2019;43:326-34.

18. Huang, Q., Wang, T. \& Wang, H. Ginsenoside Rb2 enhances the antiinflammatory effect of $\omega$-3 fatty acid in LPS-stimulated RAW264.7 macrophages by upregulating GPR120 expression. Acta Pharmacol Sin 2017; 38: 192-200. https://doi.org/https://doi.org/10.1038/aps.2016.135. 
19. Cho JY, Yoo ES, Baik KU, Park MH, Han BH. In vitro inhibitory effect of protopanaxadiol ginsenosides on tumor necrosis factor (TNF)-alpha production and its modulation by known TNF-alpha antagonists. Planta Med. 2001;67:213-8.

20. Oeckinghaus A, Ghosh S. The NF-kappaB family of transcription factors and its regulation. Cold Spring Harb Perspect Biol. 2009;1:a000034.

21. Wu CF, Bi XL, Yang JY, Zhan JY, Dong YX, Wang JH, et al. Differential effects of ginsenosides on NO and TNF-a production by LPS-activated N9 microglia. Int Immunopharmacol. 2007;7:313-20

22. Xu SY, Carlson M, Engstrom A, Garcia R, Peterson CG, Venge P. Purification and characterization of a human neutrophil lipocalin (HNL) from the secondary granules of human neutrophils. Scand J Clin Lab Invest. 1994;54:365-76.

23. Xiao R, Chen R. Neutrophil gelatinase-associated lipocalin as a potential novel biomarker for ventilator-associated lung injury. Mol Med Rep. 2017;15:3535-40.

24. Zhao P. Stephens JM. STAT1, NF-KB and ERKs play a role in the induction of lipocalin-2 expression in adipocytes. Mol Metab. 2013;2:161-70.

25. Zhao P, Elks CM, Stephens JM. The induction of lipocalin-2 protein expression in vivo and in vitro. J Biol Chem. 2014;289:5960-9.

26. Tang W, Ma J, Gu R, Ding X, Lei B, Wang X, et al. Lipocalin 2 suppresses ocular inflammation by inhibiting the activation of NF-kappabeta pathway in endotoxin-induced uveitis. Cell Physiol Biochem. 2018;46:375-88.

27. Lee JY, Linge HM, Ochani K, Lin K, Miller EJ. N-ethylmaleimide sensitive factor (NSF) inhibition prevents vascular instability following gram-positive pulmonary challenge. PLoS One. 2016;11:e0157837.

28. Davies PF. Flow-mediated endothelial mechanotransduction. Physiol Rev. 1995:75:519-60. https://doi.org/10.1152/physrev.1995.75.3.519.

29. Zhao W, He X, Hoadley KA, et al. Comparison of RNA-Seq by poly (a) capture, ribosomal RNA depletion, and DNA microarray for expression profiling. BMC Genomics. 2014;419:15.

30. Li J, Fu C, Speed TP, Wang W, Symmans WF. Accurate RNA sequencing from formalin-fixed cancer tissue to represent high-quality transcriptome from frozen tissue. JCO Precis Oncol. 2018;1:9.

31. Kwong $L N$, et al. Biological validation of RNA sequencing data from formalinfixed paraffin-embedded primary melanomas. JCO Precis Oncol. 2018:1-19.

32. Adiconis $X$, et al. Comparative analysis of RNA sequencing methods for degraded or low-input samples. Nat Methods. 2013;10:623-9.

33. Guo Y, et al. RNA sequencing of formalin-fixed, paraffin-embedded specimens for gene expression quantification and data mining. Int $J$ Genomics. 2016;2016:1-10.

\section{Publisher's Note}

Springer Nature remains neutral with regard to jurisdictional claims in published maps and institutional affiliations.

Ready to submit your research? Choose BMC and benefit from:

- fast, convenient online submission

- thorough peer review by experienced researchers in your field

- rapid publication on acceptance

- support for research data, including large and complex data types

- gold Open Access which fosters wider collaboration and increased citations

- maximum visibility for your research: over $100 \mathrm{M}$ website views per year

At $\mathrm{BMC}$, research is always in progress.

Learn more biomedcentral.com/submissions 